\title{
(CA) CLOSURES OF ANALYTIC GROUPS
}

\author{
DAVID ZERLING
}

\begin{abstract}
An analytic group $G$ is called (CA) if the group of inner automorphisms of $G$ is closed in the Lie group of all bicontinuous automorphisms of $G$. We introduce the notion of a (CA) closure for an analytic group and show that every analytic group possesses a (CA) closure. The definition of uniqueness for such a (CA) closure is developed and a sufficient condition for uniqueness is given.

We also develop new sufficient conditions for a closed normal analytic subgroup of a (CA) analytic group to be (CA).
\end{abstract}

1. Introduction. By an analytic group and an analytic subgroup of a Lie group, we mean a connected Lie group and a connected Lie subgroup, respectively. If $G$ and $H$ are Lie groups and $\phi$ is a one-to-one (continuous) homomorphism from $G$ into $H, \phi$ will be called an immersion. $\phi$ will be called closed or dense, as $\phi(G)$ is closed or dense in $H . G_{0}$ and $Z(G)$ will denote the identity component group and center of $G$, respectively.

If $G$ is an analytic group, $A(G)$ will denote the Lie group of all (bicontinuous) automorphisms of $G$, topologized with the generalized compact-open topology. $G$ will be called (CA) if $I(G)$, the Lie group of all inner automorphisms of $G$, is closed in $A(G)$. It is well known that $G$ is (CA) if and only if its universal covering group is (CA).

If $G$ is a normal analytic subgroup of an analytic group $H$, then each element $h$ of $H$ induces an automorphism of $G$, namely, $g \mapsto h g h^{-1}$. We will denote this homomorphism from $H$ into $A(G)$ by $\rho_{G H} . I_{H}(h)$ will denote the inner automorphism of $H$ determined by $h \in H$. More generally, if $A$ is a subset of $H, I_{H}(A)$ will denote the set of all inner automorphisms of $H$ determined by elements of $A$. $I_{H}(H)$ will be written as $I(H)$, and the mapping $h \mapsto I_{H}(h)$ of $H$ onto $I(H)$ will be denoted by $I_{H}$.

If $N$ is an analytic group and $H$ is an analytic subgroup of $A(N)$, then $N$ (5) will denote the semidirect product of $N$ and $H$. On the other hand, if $G$ is an analytic group containing a closed normal analytic subgroup $N$ and a closed analytic subgroup $H$, such that $G=N H, N \cap H=\{e\}$, and such that the restriction of $\rho_{N G}$ to $H$ is one-to-one, we will frequently identify $G$ with $N$ (s) $\rho_{N G}(H)$ and $H$ with $\rho_{N G}(H)$, that is, we may write $G=N$ (5) $H$.

In Zerling [6, The Main Structure Theorem], and [7, Lemma 2.11] the author proved the following theorem:

Received by the editors October 17, 1980.

1980 Mathematics Subject Classification. Primary 22E15; Secondary 22D45. 
THeOREM A. Let $G$ be a non-(CA) analytic group. Then there is a maximal (CA) closed normal analytic subgroup $M$ of $G$, a toral subgroup $T$ in $A(M)$, and a dense vector subgroup $V$ of $T$, such that:

(i) $P=M$ (3) $T$ is a (CA) analytic group.

(ii) $G$ is isomorphic to the dense analytic subgroup $M$ (s) $V$ of $P$.

(iii) $Z(G)$ is contained in $M$.

(iv) $Z_{0}(G)=Z_{0}(P)$.

(v) Each automorphism $\sigma$ of $G$ can be extended to an automorphism $\varepsilon(\sigma)$ of $P$, such that $\varepsilon: A(G) \rightarrow A(P)$ is a closed immersion.

In this paper we shall improve upon (iv) by showing that $Z(G)=Z(P)$. We shall develop a sufficient condition for a non-(CA) analytic group to possess a unique (CA) closure, as defined in $\$ 2$, and we show that each non-(CA) analytic group contains a closed non-(CA) analytic subgroup satisfying this sufficient condition. We shall also develop new sufficient conditions for a closed normal analytic subgroup of a (CA) analytic group to be (CA).

The following results of Goto will be very important to us.

Goто [2, (5.2)]. Let $L$ be an analytic group and let $N$ be a closed normal analytic subgroup of $L$. If $L / N$ is a toral group, then there is a toral group $T$ in $L$ such that $L=N T, N \cap T=\{e\}$.

Goтo [2, Theorem 2]. Let $G$ be a dense analytic subgroup of an analytic group $L$ and suppose that $G$ contains a maximal normal analytic subgroup $N$ which contains the commutator subgroup of $G$ and is also closed in $L$. Then there is a closed vector subgroup $V$ of $G$, such that $G=N V, L=N \bar{V}$, where $N \cap \bar{V}=\{e\}$ and $\bar{V}$ is a toral subgroup of $L$.

REMARK. [2, (5.2)] and the consequent Theorem 2 of Goto above are generalizations of weaker results in Goto [1]. In particular $N \cap \bar{V}$ was only shown to be finite. Since $N \cap \bar{V}=\{e\}$ now, we can improve upon (iv) of Theorem A.

2. Existence and uniqueness of (CA) closures.

Definition. Let $G$ be an analytic group. By a (CA) closure of $G$ we mean a triple $(G, f, L)$, where $L$ is a (CA) analytic group, $f: G \rightarrow L$ is a dense immersion, and $Z(f(G))=Z(L)$.

Let $G$ be a non-(CA) analytic group and let us adopt the notation of Theorem A. Let $M^{\prime}$ be a maximal analytic subgroup of $I(G)$ which contains the commutator subgroup of $I(G)$ and is closed in $A(G)$. Then from Goto [2, Theorem 2] there is a closed vector subgroup $V^{\prime}$ of $I(G)$, such that $I(G)=M^{\prime} V^{\prime}, \overline{I(G)}=M^{\prime} \bar{V}^{\prime}, M^{\prime} \cap$ $\bar{V}^{\prime}=\{e\}$, and $T^{\prime}=\bar{V}^{\prime}$ is a toral subgroup of $\overline{I(G)}$. In the proof of Theorem A we have $I_{G}(M)=M^{\prime}, I_{G}(V)=V^{\prime}$ and $\rho_{G P}(T)=T^{\prime}$, where $\rho_{G P}$ is 1-1 on $T$.

To see that $Z(G)=Z(P)$ we let $(m, \tau) \in Z(P)$ where $m \in M, \tau \in T$. Then $\rho_{G P}(m) \cdot \rho_{G P}(\tau)=e$. Therefore $\rho_{G P}(\tau)=e$ and so $\tau=e$. Hence, $Z(G)=Z(P)$. We now have the following theorem.

THEOREM 2.1. Every analytic group possesses a (CA) closure. 
Definition. Let $G$ be an analytic group. Two (CA) closures $\left(G, \psi_{1}, L_{1}\right)$ and $\left(G, \psi_{2}, L_{2}\right)$ of $G$ will be called equivalent if there exists an automorphism $\alpha$ of $G$ and an isomorphism $\beta$ from $L_{1}$ onto $L_{2}$ so that $\beta \circ \psi_{1}=\psi_{2} \circ \alpha$. We will say that $G$ possesses a unique (CA) closure if all (CA) closures of $G$ are equivalent.

Remark. From van Est [4, Theorem 2.2.1] we know that if $G$ is a dense (CA) analytic subgroup of an analytic group $L$, then $Z(L)=\overline{Z(G)}, L=G \cdot \overline{Z(G)}$, and $L$ is also (CA). Hence, it is clear that each (CA) analytic group has a unique (CA) closure, namely itself.

THeORem 2.2. Let $G$ be a (CA) analytic group and let $N$ and $H$ be a closed normal analytic subgroup and a closed analytic subgroup of $G$, respectively, such that $G=N H, N \cap H=\{e\}$. Let $\pi$ denote the natural projection of $G$ onto $H$. If $\pi(Z(G))$ is closed in $H$, then $N$ is (CA).

Proof. Suppose that $N$ is non-(CA). Let $N^{\prime}$ be a (CA) analytic group containing $N$ as a dense subgroup, where $N^{\prime}$ is to be constructed according to Theorem $\mathrm{A}$. From Theorem 2.1 we know $Z(N)=Z\left(N^{\prime}\right)$. Let $\varepsilon: A(N) \rightarrow A\left(N^{\prime}\right)$ be the extension homomorphism of Theorem A. Let $\beta=\varepsilon \circ \rho_{N G}$. Then the restriction of $\beta$ to $H$ is a homomorphism of $H$ into $A\left(N^{\prime}\right)$, and we let $G^{\prime}$ denote the semidirect product of $N^{\prime}$ and $H$ that is determined by $\beta$. Then $G$ is dense in $G^{\prime}$.

Let $\left\{\left(n_{k}, h_{k}\right)\right\}$ be a sequence of central elements in $G$ converging in $G^{\prime}$ to $\left(n^{\prime}, h\right)$. Since $\pi(Z(G))$ is closed in $H$, there exists an element $\bar{n}$ in $N$ so that $(\bar{n}, h)$ is in $Z(G)$. Since $n^{\prime} \bar{n}^{-1}=\left(n^{\prime}, h\right) \cdot(\bar{n}, h)^{-1}$, we see that $n^{\prime} \bar{n}^{-1}$ is in $Z\left(G^{\prime}\right) \cap N^{\prime}$. Therefore, $n^{\prime} \bar{n}^{-1}$ is in $Z\left(N^{\prime}\right)$. Since $Z(N)=Z\left(N^{\prime}\right), n^{\prime} \bar{n}^{-1}$ is in $Z(N)$. Therefore, since $n^{\prime} \bar{n}^{-1}$ is already in $Z\left(G^{\prime}\right)$, it follows that $n^{\prime} \bar{n}^{-1} \in Z(G)$. So $\left(n^{\prime}, h\right)=z \cdot(\bar{n}, h), z \in Z(G)$. So $Z(G)$ is closed in $G^{\prime}$. Since $G$ is (CA), $G=G^{\prime}$ by van Est [4, Theorem 2.2.1]. Hence $N=N^{\prime}$. Therefore $N$ is (CA). Q.E.D.

COROllary. Let $G$ be a (CA) analytic group and let $N$ be a closed normal analytic subgroup of $G$. If (i) $Z(G) \cap N$ is a uniform subgroup of $Z(G)$, and (ii) $G / N$ is a toral group, then $N$ is (CA).

Proof. From Goto [2] there is a toral group $T$ of $G$ such that $G=N \cdot T$, $N \cap T=\{e\}$. Since $Z(G) \cap N$ is uniform in $Z(G), \pi(Z(G))$ is compact, where $\pi$ is the natural projection of $G$ onto $T$. Therefore, $N$ is (CA) from Theorem 2.2. Q.E.D.

Theorem 2.3. Adopting the notation of Theorem A let $G=M V$ be a non-(CA) analytic group. Let $f: G \rightarrow L$ be a dense immersion of $G$ into a (CA) analytic group $L$. Suppose $Z(f(G))$ is a uniform subgroup of $Z(L)$. Then there is a closed vector subgroup $W$ of $G$ such that $G=M W, L=f(M) \cdot \overline{f(W)}, f(M) \cap \overline{f(W)}=\{e\}$, and $\overline{f(W)}$ is a toral group.

Proof. From Theorem 2.1 of Zerling [7] we know that $\overline{f(M)}=f(M) \cdot \overline{f(Z(G))}$. Therefore $f(M)$ is closed in $L$. Let $J$ be a maximal analytic subgroup of $G$, which contains $M$ and for which $f(J)$ is closed in $L$. Let $\pi$ denote the natural projection of $J$ on $V$. Then $J=M U$, where $U=\pi(J)$. 
From Goto [2, Theorem 2] there is a closed vector subgroup $W$ of $G$ so that $G=J \cdot W, L=f(J) \cdot \overline{f(W)}, f(J) \cap \overline{f(W)}=\{e\}$, and $\overline{f(W)}$ is a toral group. Since $Z(f(G))$ is contained in $f(M)$ and is uniform in $Z(L), \pi^{\prime}(Z(L))$ is compact in $\overline{f(W)}$, where $\pi^{\prime}$ is the natural projection of $L$ onto $\overline{f(W)}$. By Theorem $2.2, J$ is (CA), since $L$ is (CA). But $M$ is a maximal (CA) closed normal analytic subgroup of $G$ from Theorem A. Therefore, $J=M$. Q.E.D.

THEOREM 2.4. Let $G$ be a non-(CA) analytic group and suppose that $G / Z(G)$ is homeomorphic to Euclidean space. Then $G$ has a unique (CA) closure.

Proof. Let $f: G \rightarrow P$ be the dense immersion of Theorem 2.1. That is, $G=M V$ $\cong M$ (5) $\rho_{M G}(V), \quad P=M$ (5) $\overline{\rho_{M G}(V)}$, and $f: G \rightarrow P$ is given by $f(m v)=$ $\left(m, \rho_{M G}(v)\right)$. From the convention in the Introduction we will write $G=M$ (ब) $V$ and $P=M$ (5) $\overline{f(V)}$. Let $(G, \psi, L)$ also be a (CA) closure of $G$. We will show that $(G, f, P)$ is equivalent to $(G, \psi, L)$.

Since $Z(\psi(G))=Z(L)$, we know from Theorem 2.3 that there is a closed vector subgroup $W$ of $G$ so that $G=M W, L=\psi(M) \cdot \overline{\psi(W)}, \psi(M) \cap \overline{\psi(W)}=\{e\}$, and $\overline{\psi(W)}$ is a toral group. Since $Z(L)=Z(\psi(G)) \subset \psi(M), \rho_{G L}$ is $1-1$ on $\overline{\psi(W)}$. Therefore $L=\psi(M)$ (s) $\overline{\psi(W)}$.

Let $M^{\prime}=I_{G}(M), W^{\prime}=I_{G}(W)$, and $V^{\prime}=I_{G}(V)$. We see that

$$
\overline{I(G)}=M^{\prime} \cdot \rho_{G P} \overline{(f(V))}=M^{\prime} \bar{V}^{\prime}, \quad M^{\prime} \cap \bar{V}^{\prime}=\{e\} .
$$

But $\overline{I(G)}=M^{\prime} \cdot \rho_{G L} \overline{(\psi(W))}=M^{\prime} \cdot \bar{W}^{\prime}, M^{\prime} \cap \bar{W}^{\prime}=\{e\}$. Therefore, $\bar{V}^{\prime}$ and $\bar{W}^{\prime}$ are each maximal toral subgroups of $\overline{I(G)}$. Hence, there is an element $\gamma$ of $\overline{I(G)}$ so that $\bar{W}^{\prime}=\gamma \bar{V}^{\prime} \gamma^{-1}$. But $M$ is $\gamma$-stable. Therefore $\left.\gamma\right|_{M} \in A(M)$.

Now $\gamma V^{\prime} \gamma^{-1} \subset I(G)=M^{\prime} W^{\prime}$, since $I(G)$ is normal in $\overline{I(G)}$. On the other hand $\gamma V^{\prime} \gamma^{-1} \subset \overline{W^{\prime}}$ and $\overline{W^{\prime}} \cap M^{\prime}=\{e\}$. Hence $\gamma V^{\prime} \gamma^{-1}=W^{\prime}$. Consequently $\left.\gamma\right|_{M} \cdot \overline{f(V)}$. $\left.\gamma\right|_{M} ^{-1}=\overline{\psi(W)}$ and $\left.\left.\gamma\right|_{M} \cdot f(V) \cdot \gamma\right|_{M} ^{-1}=\psi(W)$.

Let $\beta: P \rightarrow L$ be given by

$$
\beta(m, \tau)=\left(\psi(\gamma(m)),\left.\left.\gamma\right|_{M} \cdot \tau \cdot \gamma\right|_{M} ^{-1}\right), \quad m \in M, \tau \in \overline{f(V)} .
$$

Then $\beta$ is an isomorphism of $P$ onto $L$, and since $\rho_{M G}(\gamma(v))=\left.\left.\gamma\right|_{M} \cdot \rho_{M G}(v) \cdot \gamma\right|_{M} ^{-1}$, we see that $\beta \circ f=\psi \circ \gamma$. Hence, $(G, f, P)$ is equivalent to $(G, \psi, L)$. Q.E.D.

REMARK. Since a semisimple analytic subgroup of the general linear group possesses a nontrivial compact subgroup, we see that the condition of Theorem 2.4 that $G / Z(G)$ is homeomorphic to Euclidean space implies that $G$ is solvable.

\section{Abundance of groups with unique (CA) closure.}

LemMA 3.1. Let $G=M V$ be a non-(CA) analytic group as in Theorem A.

(i) If $Z(M)$ is connected, then $Z(G)$ is connected.

(ii) If $M / Z(M)$ is homeomorphic to Euclidean space, then $G / Z(G)$ is homeomorphic to Euclidean space.

Proof. (i) Since $Z(M)$ is a connected abelian group containing $Z(G)$ we let $S$ be a minimal abelian analytic subgroup of $G$ which contains $Z(G)$ and is contained in $Z(M)$. From Goto [2, (7.2) and (8.1)] there is a closed vector subgroup $W$ of $G$ 
such that $G=M W, M \cap W=\{e\}$, and a closed abelian analytic subgroup $H$ of $G$ (called a $g m$-torus of $G$ ) such that $H$ contains both $S$ and $W$. (The existence of $H$ containing $S$ follows from Goto's (8.1) and the existence of $W$, for such an $H$, follows from Goto's (7.2)). Therefore, $S$ commutes with each element of $M$ and $W$, i.e., $S=Z(G)$. Thus, $Z(G)$ is connected.

(ii) Since $M / Z(M)$ is homeomorphic to Euclidean space, $Z(M)$ and, therefore, $Z(G)$ are connected. Since $M / Z(M)=(M / Z(G)) /(Z(M) / Z(G))$, we can show that $M / Z(G)$ is homeomorphic to Euclidean space, if we can show that $Z(M) / Z(G)$ is a vector group.

To this end we will show that $Z(G)$ contains the maximal toral subgroup of $Z(M)$. $V$ acts on $Z(M)$ via $z \mapsto v z v^{-1}, z \in Z(M), v \in V$. Let $K$ be the maximal toral subgroup of $Z(M)$. Then $v K v^{-1}=K$ for all $v \in V$. Since $V$ is connected $v k v^{-1}=k$ for all $v \in V, k \in K$. Therefore, each $k \in K$ commutes with the elements of $M$ and $V$, i.e., $K \subset Z(G)$. Hence $M / Z(G)$ is homeomorphic to Euclidean space.

Since $M / Z(G)$ contains all of the maximal compact subgroups of $G / Z(G)$, we see that $G / Z(G)$ is homeomorphic to Euclidean space. Q.E.D.

LEMMA 3.2. Let $N$ be a nilpotent analytic group and let $V$ be a vector subgroup of $A(N)$, such that $\bar{V}$ is a toral group. Then

(i) $G=N$ (3) $V$ is non-(CA).

(ii) $\hat{G}=N$ @ $\bar{V}$ is (CA) and $Z(\hat{G})=Z(G) \subset N$.

(iii) $\overline{I(G)}=I_{G}(N) \cdot \overline{I_{G}(V)}$, where $\overline{I_{G}(V)}$ is a toral group, $I_{G}(N) \cap \overline{I_{G}(V)}=\{e\}$ and $I(G)=I_{G}(N) \cdot I_{G}(V)$, where $I_{G}(V)$ is a vector subgroup of $\overline{I_{G}(V)}$.

Proof. Since $N$ is nilpotent and therefore (CA), $I(N)$ is a closed subgroup of $A(N)$, which is homeomorphic to Euclidean space. Hence, $\bar{V} \cap I(N)=\{e\}$. This implies that the center of $N$ (s) $\bar{V}$ is contained in $N$. Therefore, $G=N$ (5) $V$ is dense in $\hat{G}=N$ (5) $\bar{V}$ with $Z(G)=Z(\hat{G})$. Hence, $G$ is non-(CA) by van Est [4].

Since $N$ is nilpotent, $I_{G}(N)$ and $I_{\hat{G}}(N)$ are closed in $A(G)$ and $A(\hat{G})$, resp. Therefore, $\hat{G}$ is (CA) since $I_{\hat{G}}(\bar{V})$ is compact. Also $\overline{I(G)}=I_{G}(N) \cdot \overline{I_{G}(V)}$, since $\overline{I_{G}(V)}=I_{G}(\bar{V})$ is a toral group.

Since $Z(\hat{G}) \subset N, I_{G}(N) \cap \overline{I_{G}(V)}=\{e\}$, and $I_{G}(V)$ is a vector subgroup of $\overline{I_{G}(V)}$. (Consequently, $I_{G}(N)$ is a maximal analytic subgroup of $I(G)$ which contains the commutator subgroup of $I(G)$ and is closed in $A(G)$.) Q.E.D.

THEOREM 3.1. Every non-(CA) analytic group $L$ contains a closed non-(CA) analytic subgroup $G$ such that $G / Z(G)$ is homeomorphic to Euclidean space (and therefore $G$ has a unique (CA) closure). Moreover, if $L$ is solvable, then $G$ is normal in $L$.

Proof. Since $L$ is non-(CA), the radical of $L, R$, is also non-(CA) from van Est [5, Theorem 2a]. Let $R=M V$ be the decomposition of Theorem $\mathrm{A}$, and let $M$ denote the closure of the commutator subgroup of $R$. Since $R$ is solvable, $N$ is nilpotent and therefore (CA). From Zerling [6, Theorem 3.2] $V$ acts effectively on $N$. Since the closure of $I_{R}(V)$ in $A(R)$ is a toral group, and since $N$ is characteristic 
in $R$, we see that the closure of $\rho_{N R}(V)$ in $A(N)$ is a toral group. Hence, Lemma 3.2 shows that $G=N V$ is a non-(CA) closed normal analytic subgroup of $R$. We want to show that $G=N$ (5) $V$ is the " $M$ (5) $V$ " type decomposition of Theorem A. However, this is an immediate result of Lemma 3.2.

Hence, since $N$ is nilpotent, $N / Z(N)$ is homeomorphic to Euclidean space. Therefore, $G / Z(G)$ is homeomorphic to Euclidean space from Lemma 3.1. Q.E.D.

REMARK. In Theorem 3.1 we simply wanted to show that every non-(CA) analytic group contains some non-(CA) analytic subgroup possessing a unique (CA) closure. The relationship between $G$ and $L$ actually exists in the relationship between $G$ and $R$, the radical of $L$. This relationship is discussed in greater detail (including some open questions) in Stevens [3].

CONJECTURE. In the proof of Theorem 2.4 we were able to show that $\bar{W}^{\prime}=$ $\gamma \bar{V}^{\prime} \gamma^{-1}, \gamma \in \overline{I(G)}$, only because we knew $\overline{V^{\prime}}$ and $\overline{W^{\prime}}$ were each maximal toral subgroups of $\overline{I(G)}$. However, they were assured of being maximal toral subgroups only because $M^{\prime}$ was homeomorphic to Euclidean space due to our hypothesis that $G / Z(G)$ is homeomorphic to Euclidean space.

The author conjectures that every non-(CA) analytic group possesses a unique (CA) closure. The existence of the above $\gamma$ and, therefore, of the unique (CA) closure would still be assured without knowing that $M^{\prime}$ was homeomorphic to Euclidean space, if we were able to prove the following: Let $L$ be an analytic subgroup of $G L(n, \mathbf{R})$ and let $M$ be a closed normal analytic subgroup of $\bar{L}$. Suppose $V$ and $W$ are each closed vector subgroups of $L$ such that (i) $L=M V=$ $M W$, and (ii) $\bar{L}=M \bar{V}=M \bar{W}, M \cap \bar{V}=M \cap \bar{W}=\{e\}$, where $\bar{V}$ and $\bar{W}$ are toral groups. Then there exists $\gamma \in \bar{L}$ such that $\gamma \bar{V} \gamma^{-1}=\bar{W}$.

\section{REFERENCES}

1. M. Goto, Analytic subgroups of $G L(n, R)$, Tôhoku Math. J. (2) 25 (1973), 197-199.

2. ___ Immersions of Lie groups, J. Math. Soc. Japan (to appear).

3. T. C. Stevens, Weakened topology for Lie groups, $\mathrm{Ph}$. D. Thesis, Dept. of Math., Harvard Univ., Cambridge, Mass., 1978.

4. W. T. van Est, Dense imbeddings of Lie groups, Indag. Math. 14 (1952), 255-274.

5. __ Some theorems on (CA) Lie algebras. I, II, Indag. Math. 14 (1952), 546-568.

6. D. Zerling, Some theorems on (CA) analytic groups, Trans. Amer. Math. Soc. 205 (1975), 181-192.

7. __ Dense subgroups of Lie groups. II, Trans. Amer. Math. Soc. 246 (1978), 419-428.

Department of Mathematics and Physics, Philadelphia College of Textiles and Science, Philladelphila, Pennsylvania 19144 